PROCEEDINGS OF THE AMERICAN MATHEMATICAL SOCIETY

Volume 125, Number 3, March 1997, Pages 839-848

S 0002-9939(97)03642-3

\title{
AN EMBEDDING THEOREM OF SOBOLEV TYPE FOR AN OPERATOR WITH SINGULARITY
}

\author{
SHUJI WATANABE
}

(Communicated by Palle E. T. Jorgensen)

\begin{abstract}
We discuss spaces of Sobolev type which are defined by the operator with singularity: $\mathcal{D}=d / d x-(c / x) R$, where $R u(x)=u(-x)$ and $c>1$. This operator appears in a one-dimensional harmonic oscillator governed by Wigner's commutation relations. We study smoothness of $u$ and continuity of $u / x^{\beta}(\beta>0)$ where $u$ is in each space of Sobolev type, and obtain a generalization of the Sobolev embedding theorem. On the basis of a generalization of the Fourier transform, the proof is carried out. We apply the result to the Cauchy problems for partial differential equations with singular coefficients.
\end{abstract}

\section{INTRODUCTION AND THE MAIN RESULT}

This paper is concerned with spaces of Sobolev type defined by the operator with singularity:

$$
\mathcal{D}=\frac{d}{d x}-\frac{c}{x} R, \quad R u(x)=u(-x),
$$

with a real constant $c$ specified later. The operator $p=-i \mathcal{D}$, called the momentum operator, appears in a one-dimensional harmonic oscillator governed by Wigner's commutation relations [10]: $i p=[x, H]$ and $-i x=[p, H]$, where the Hamiltonian is of the classical form $H=\left(p^{2}+x^{2}\right) / 2$. Assuming that $x$ is the multiplication by $x$, Yang [11] derived the above expression for $\mathcal{D}$. When $c=0$, Wigner's commutation relations are reduced to the canonical commutation relations in quantum mechanics and $\mathcal{D}$ becomes the differential operator $d / d x$.

Self-adjointness of the momentum operator $p$ and of the Hamiltonian $H$ is established (see [3] and [8]). On the basis of the Bessel transform $B$, the spectrum of the self-adjoint operator $p$ is studied in [3]. This transform is a unitary operator from $L^{2}\left(\mathbf{R}^{1}\right)$ onto itself [3, Theorem 3.2]:

$$
(B u, B v)=(u, v), \quad u, v \in L^{2}\left(\mathbf{R}^{1}\right),
$$

where $(\cdot, \cdot)$ denotes the inner product of $L^{2}\left(\mathbf{R}^{1}\right)$ defined by

$$
(u, v)=\int_{-\infty}^{\infty} u(x) \overline{v(x)} d x .
$$

Received by the editors September 22, 1995.

1991 Mathematics Subject Classification. Primary 35G10, 46E35, 47B25.

Key words and phrases. Embedding theorem of Sobolev type, operator with singularity, partial differential equations with singular coefficients.

Research partially supported by Grant-in-Aid for Scientific Research (No. 07740175), Ministry of Education, Science, Sports and Culture. 
Moreover, we have

$$
\begin{aligned}
& B u(y)=\text { l. i.m. } L \rightarrow \infty \int_{-L}^{L} \overline{\varphi(x y)} u(x) d x, \\
& B^{*} u(x)=\text { l. i. m. } L \rightarrow \infty \int_{-L}^{L} \varphi(x y) u(y) d y
\end{aligned}
$$

for $u \in L^{2}\left(\mathbf{R}^{1}\right)[3$, Theorem 3.4]. Here

$$
\varphi(x y)=\frac{\sqrt{|x y|}}{2}\left\{J_{c-1 / 2}(|x y|)+i \operatorname{sgn}(x y) J_{c+1 / 2}(|x y|)\right\}
$$

and $J_{\nu}$ is a Bessel function. If $c=0$, then $\varphi(x y)=e^{i x y} / \sqrt{2 \pi}$ and hence the Bessel transform coincides with the Fourier transform. For this reason the Bessel transform can be regarded as a generalization of the Fourier transform. Ohnuki and Kamefuchi [2, pp.289-296] were the first to obtain the function $\varphi$ in the study of the eigenvalue problem of the momentum operator $p$ :

$$
p \varphi=y \varphi .
$$

The multiplication by $y$ is self-adjoint on the set $D(y)=\left\{u(y): u, y u \in L^{2}\left(\mathbf{R}^{1}\right)\right\}$. With the aid of the Bessel transform, the self-adjont operators $p$ and $y$ are shown to be unitarily equivalent to each other [3, Proposition 4.2]:

$$
y=B p B^{*} .
$$

For unitary equivalence, see e.g. Goldstein [1, p. 94].

We now define spaces of Sobolev type using the Bessel transform $B$ :

\section{Definition 1.1.}

$$
\mathcal{H}^{m}\left(\mathbf{R}^{1}\right)=\left\{u \in L^{2}\left(\mathbf{R}^{1}\right): \int_{-\infty}^{\infty}\left(1+y^{2}\right)^{m}|B u(y)|^{2} d y<\infty\right\},
$$

where $m=0,1,2, \cdots$.

The space of Sobolev type $\mathcal{H}^{m}\left(\mathbf{R}^{1}\right)$ is a Hilbert space with inner product

$$
(u, v)_{m}=\int_{-\infty}^{\infty}\left(1+y^{2}\right)^{m} B u(y) \overline{B v(y)} d y
$$

and norm $|u|_{m}=(u, u)_{m}^{1 / 2}$.

Remark. The Bessel transform $B$ depends on the constant $c$, and so does $\mathcal{H}^{m}\left(\mathbf{R}^{1}\right)$. When $c=0, B$ coincides with the Fourier transform and $\mathcal{H}^{m}\left(\mathbf{R}^{1}\right)$ turns out to be the Sobolev space $H^{m}\left(\mathbf{R}^{1}\right)$.

We introduce the following spaces. For each $m=0,1,2, \cdots$, let $\mathcal{B}^{m}\left(\mathbf{R}^{1}\right)=\left\{u \in C^{m}\left(\mathbf{R}^{1}\right): \frac{d^{k} u}{d x^{k}}(k=0,1, \cdots, m)\right.$ are bounded on $\left.\mathbf{R}^{1}\right\}$, $\mathcal{F}^{m}\left(\mathbf{R}^{1}\right)=\left\{u(x): u, \frac{u}{x^{k}}(k=1,2, \cdots, m)\right.$ are continuous and bounded on $\left.\mathbf{R}^{1}\right\}$.

As is well known, $\mathcal{B}^{m}\left(\mathbf{R}^{1}\right)$ is a Banach space with norm

$$
|u|_{\mathcal{B}^{m}}=\max _{k=0,1, \cdots, m}\left\{\sup _{x \in \mathbf{R}^{1}}\left|\frac{d^{k} u}{d x^{k}}(x)\right|\right\} .
$$


The space $\mathcal{F}^{m}\left(\mathbf{R}^{1}\right)$ is a Banach space with norm

$$
|u|_{\mathcal{F}^{m}}=\max _{k=0,1, \cdots, m}\left\{\sup _{x \in \mathbf{R}^{1}}\left|\frac{u(x)}{x^{k}}\right|\right\},
$$

as is shown below (see Lemma 2.1).

The purpose of this paper is to prove the following theorem, and then to apply it to the Cauchy problems for partial differential equations with singular coefficients.

Theorem 1.2. Suppose $c>1$. Then, for each $u \in \mathcal{H}^{m}\left(\mathbf{R}^{1}\right)$, there is an element $v \in \mathcal{B}^{\alpha}\left(\mathbf{R}^{1}\right) \cap \mathcal{F}^{\beta}\left(\mathbf{R}^{1}\right)$ such that $u(x)=v(x) \quad$ (a.a. $\left.x \in \mathbf{R}^{1}\right)$, i.e.,

$$
\mathcal{H}^{m}\left(\mathbf{R}^{1}\right) \subset \mathcal{B}^{\alpha}\left(\mathbf{R}^{1}\right) \cap \mathcal{F}^{\beta}\left(\mathbf{R}^{1}\right)
$$

where

$$
\alpha= \begin{cases}m-1 & (c=2 k), \\ \min (m-1, c-1) & (c=2 k+1) \\ \min (m-1,[c]) & (\text { otherwise })\end{cases}
$$

and

$$
\beta= \begin{cases}\min (m-1, c) & (c=2 k) \\ \min (m-1, c-1) & (c=2 k+1) \\ \min (m-1,[c]) & (\text { otherwise })\end{cases}
$$

with $k=1,2,3, \cdots$. Moreover, $\quad|v|_{\mathcal{B}^{\alpha}} \leq a|u|_{m} \quad$ and $\quad|v|_{\mathcal{F}^{\beta}} \leq b|u|_{m}$, where $a$ and $b$ are positive constants and depend on $m$ and $c$ only.

Remark. The relation $\alpha=m-1$ holds not only for $c=0$ (the Sobolev embedding theorem) but also for $c=2,4,6, \cdots$.

In [9] the following result is obtained : Let $c>1$ and let $\Omega$ be a bounded open interval of $\mathbf{R}^{1}$. Then, for each $u \in \mathcal{H}^{m}\left(\mathbf{R}^{1}\right)$, there is an element $v \in C^{\alpha}(\Omega)$ such that $u(x)=v(x)$ (a.a. $x \in \Omega$ ) and $v / x^{\beta}$ is continuous on $\Omega$, where $\alpha$ and $\beta$ are those in Theorem 1.2. However, $\mathcal{H}^{m}\left(\mathbf{R}^{1}\right)$ is defined in another way [9], and it is expected that the two definitions are equivalent to each other.

\section{Preliminaries}

We begin this section by showing that $\mathcal{F}^{m}\left(\mathbf{R}^{1}\right)$ is a Banach space.

Lemma 2.1. The space $\mathcal{F}^{m}\left(\mathbf{R}^{1}\right)$ is a Banach space with norm

$$
|u|_{\mathcal{F}^{m}}=\max _{k=0,1, \cdots, m}\left\{\sup _{x \in \mathbf{R}^{1}}\left|\frac{u(x)}{x^{k}}\right|\right\} .
$$

Proof. It suffices to show that $\mathcal{F}^{m}\left(\mathbf{R}^{1}\right)$ is complete. Let $\left\{u_{n}\right\}$ be a Cauchy sequence in $\mathcal{F}^{m}\left(\mathbf{R}^{1}\right)$. Then $\left\{u_{n}\right\},\left\{u_{n} / x^{k}\right\}$ converge uniformly on $\mathbf{R}^{1}$ to functions $u, u_{(k)} \quad(k=1,2, \cdots, m)$, respectively. Hence $u, u_{(k)}$ are continuous on $\mathbf{R}^{1}$. Since

$u_{n}, u_{n} / x^{k}$ are bounded on $\mathbf{R}^{1}$, it follows that $u, u_{(k)}$ are also bounded on $\mathbf{R}^{1}$. Moreover, $u(x) / x^{k}=u_{(k)}(x) \quad(k=1,2, \cdots, m)$ at $x \neq 0$. Note that $u_{(k)}(x) \rightarrow u_{(k)}(0)$ as $x \rightarrow 0$. Let $\left[u / x^{k}\right]_{x=0}=u_{(k)}(0)(k=1,2, \cdots, m)$. Then, at all $x \in \mathbf{R}^{1}$,

$$
\frac{u(x)}{x^{k}}=u_{(k)}(x) \quad(k=1,2, \cdots, m) \text {. }
$$

Thus, $u \in \mathcal{F}^{m}\left(\mathbf{R}^{1}\right)$ and $\left|u-u_{n}\right|_{\mathcal{F}^{m}} \rightarrow 0$ as $n \rightarrow \infty$. Hence $\mathcal{F}^{m}\left(\mathbf{R}^{1}\right)$ is complete.

From Definition 1.1 we immediately obtain the following. 
Lemma 2.2. (A) $\mathcal{H}^{0}\left(\mathbf{R}^{1}\right)=L^{2}\left(\mathbf{R}^{1}\right)$.

(B) $\mathcal{H}^{m^{\prime}}\left(\mathbf{R}^{1}\right) \subset \mathcal{H}^{m}\left(\mathbf{R}^{1}\right), \quad m^{\prime} \geq m$.

(C) $|u|_{m} \leq|u|_{m^{\prime}}, \quad u \in \mathcal{H}^{m^{\prime}}\left(\mathbf{R}^{1}\right), \quad m^{\prime} \geq m$.

Definition 2.3. We define $\mathcal{D}=d / d x-(c / x) R: \mathcal{H}^{1}\left(\mathbf{R}^{1}\right) \rightarrow L^{2}\left(\mathbf{R}^{1}\right)$ by

$$
\mathcal{D} u=i B^{*} y B u, \quad u \in \mathcal{H}^{1}\left(\mathbf{R}^{1}\right),
$$

where $B$ is the Bessel transform and $y$ the multiplication by $y$ (see also (1.5)).

Lemma 2.4. (A) The domain of the operator $\mathcal{D}^{m}(m=1,2, \cdots)$ coincides with $\mathcal{H}^{m}\left(\mathbf{R}^{1}\right)$, i.e., $D\left(\mathcal{D}^{m}\right)=\mathcal{H}^{m}\left(\mathbf{R}^{1}\right)$.

(B) $\mathcal{H}^{m}\left(\mathbf{R}^{1}\right)=B^{*} D\left(y^{m}\right)$.

(C) The operator $\mathcal{D}$ is skew-adjoint on $\mathcal{H}^{1}\left(\mathbf{R}^{1}\right)$.

Proof. (A) and (B) are immediate consequences of Definitions 1.1 and 2.3. (C) follows since the Bessel transform $B$ is unitary (see (1.1)) and the multiplication $y$ is self-adjoint.

The following is our key lemma, which plays an essential role throughout this paper.

Lemma 2.5. Let $u \in \mathcal{H}^{m}\left(\mathbf{R}^{1}\right)$ and let $k=0,1,2, \cdots, m-1$. Then $y^{k} B u \in$ $L^{1}\left(\mathbf{R}^{1}\right)$.

Proof. By the Schwarz inequality,

$$
\int_{-\infty}^{\infty}\left|y^{k} B u(y)\right| d y \leq\left\{\int_{-\infty}^{\infty} \frac{y^{2 k}}{\left(1+y^{2}\right)^{m}} d y\right\}^{1 / 2}|u|_{m}<\infty .
$$

The lemma follows.

For later convenience we enumerate some properties of the function $\varphi$ given by (1.3).

$$
\varphi(z)= \begin{cases}O\left(|z|^{c}\right) & (z \rightarrow 0) \\ O(\cos z) & (|z| \rightarrow \infty)\end{cases}
$$

Let $c>1$. Then

$$
|\varphi(z)| \leq \text { constant } \cdot|z|^{c} \text { for } z \text { in each bounded interval of } \mathbf{R}^{1} ;
$$

where

$$
\varphi(\cdot) \in C^{\gamma}\left(\mathbf{R}^{1}\right),
$$

$$
\gamma= \begin{cases}\infty & (c=2 k) \\ c-1 & (c=2 k+1) \\ {[c]} & \text { (otherwise) }\end{cases}
$$

with $k=1,2,3, \cdots$. 


\section{Proof of the MAIN RESUlt}

We prove our main result, Theorem 1.2, in a sequence of lemmas. We now turn to $(1.2)$. Let $c>1$. For each $u \in \mathcal{H}^{m}\left(\mathbf{R}^{1}\right)$,

$$
u(x)=\lim _{n \rightarrow \infty} \int_{-n}^{n} \varphi(x y) B u(y) d y \quad\left(\text { a.a. } x \in \mathbf{R}^{1}\right) .
$$

(2.1) implies that the function $y \mapsto\left|\varphi(x y) / \sqrt{1+y^{2}}\right|^{2}$ is integrable on $\mathbf{R}^{1}$. So is the function $y \mapsto \varphi(x y) B u(y)$. Hence

$$
u(x)=\int_{-\infty}^{\infty} \varphi(x y) B u(y) d y \quad\left(\text { a.a. } x \in \mathbf{R}^{1}\right) .
$$

Set

$$
v(x)=\int_{-\infty}^{\infty} \varphi(x y) B u(y) d y .
$$

Then $u(x)=v(x) \quad$ (a.a. $\left.x \in \mathbf{R}^{1}\right)$. We will see below that this function $v$ is nothing but the element $v$ mentioned in Theorem 1.2 In what follows, we study smoothness of $v$ together with continuity of $v / x^{\beta}$.

Lemma 3.1. Let $c$ and $\alpha$ be as in Theorem 1.2. Then $v$ is $\alpha$ times differentiable on $\mathbf{R}^{1}$.

Proof. Combining (1.4) with (2.3) yields

$$
\frac{\partial \varphi}{\partial x}=\left(i \varphi+\frac{c}{x y} R \varphi\right) y \quad \text { and } \quad \frac{\partial^{\alpha} \varphi}{\partial x^{\alpha}}=\left\{\sum_{k=0}^{\alpha} \frac{a_{k}^{(\alpha)} \varphi+b_{k}^{(\alpha)} R \varphi}{(x y)^{\alpha-k}}\right\} y^{\alpha}
$$

with $a_{k}^{(\alpha)}$ and $b_{k}^{(\alpha)}$ chosen appropriately. We formally differentiate $v \quad \alpha$ times:

$$
\frac{d^{\alpha} v}{d x^{\alpha}}=\int_{-\infty}^{\infty} \frac{\partial^{\alpha} \varphi}{\partial(x y)^{\alpha}} y^{\alpha} B u(y) d y
$$

The result follows from (2.1), (2.3) and Lemma 2.5.

An elementary calculation gives the following lemma.

Lemma 3.2. Suppose that $g: \mathbf{R}^{1} \times \mathbf{R}^{1} \rightarrow \mathbf{C}^{1}$ satisfies the following conditions.

(i) $|g(x, y)| \leq$ constant for all $x, y \in \mathbf{R}^{1}$.

(ii) For each $L>0$, there are positive numbers $K, \beta$ such that

$$
|g(x, y)-g(a, y)| \leq K|x-a|^{\beta}, \quad \text { where }|y| \leq L .
$$

Then the function $F$ defined by

$$
F(x)=\int_{-\infty}^{\infty} g(x, y) f(y) d y \quad\left(f \in L^{1}\left(\mathbf{R}^{1}\right)\right)
$$

is continuous at $x=a$.

Lemma 3.3. Let $c$ and $\alpha$ be as in Theorem 1.2. Let $v$ be given by (3.1).

(A) $v \in C^{\alpha}\left(\mathbf{R}^{1}\right)$.

(B) $\quad v \in \mathcal{B}^{\alpha}\left(\mathbf{R}^{1}\right)$ and $|v|_{\mathcal{B}^{\alpha}} \leq a|u|_{m}$, where $a$ is a positive constant and depends on $m$ and $c$ only. 
Proof. (A) We need to show the continuity of $d^{\alpha} v / d x^{\alpha}$ (see Lemma 3.1).

The case where $c=2,4,6, \cdots$. Note that $\alpha=m-1$. Thanks to Lemma 2.5, we have only to show that $\partial^{\alpha} \varphi / \partial(x y)^{\alpha}$ in (3.2) satisfies conditions (i) and (ii) of Lemma 3.2. But this immediately follows from (2.1) and (2.3).

The other cases. It follows from (2.3), Lemma 2.5 and Lemma 3.2 that the functions

$$
x \mapsto \int_{-\infty}^{\infty} \varphi(x y) y^{k} B u(y) d y \text { and } x \mapsto \int_{-\infty}^{\infty} R \varphi(x y) y^{k} B u(y) d y
$$

$(k=0,1,2, \cdots, \alpha)$ are continuous at $x \neq 0$. Thus $d^{\alpha} v / d x^{\alpha}$ is shown to be continuous at $x \neq 0$. So it remains to show the continuity at $x=0$. Note that $d^{\alpha} v / d x^{\alpha}(0)=0 . \quad \partial^{\alpha} \varphi / \partial(x y)^{\alpha}$ clearly satisfies condition (i) of Lemma 3.2 by (2.1). Moreover, (2.2) gives

$$
\left|\frac{\partial^{\alpha} \varphi(x y)}{\partial(x y)^{\alpha}}\right| \leq \text { constant } \cdot \sum_{k=0}^{\alpha}\left(\left|a_{k}^{(\alpha)}\right|+\left|b_{k}^{(\alpha)}\right|\right)|x y|^{c-\alpha+k},
$$

where $c-\alpha+k>0$. Therefore, condition (ii) of Lemma 3.2 is also satisfied. Hence $d^{\alpha} v / d x^{\alpha}$ is continuous at $x=0$.

(B) From (3.2) we see that for $k=0,1,2, \cdots, \alpha$,

$$
\begin{aligned}
\left|\frac{d^{k} v}{d x^{k}}(x)\right| \leq & \left\{\int_{-\infty}^{\infty} \frac{1}{1+y^{2}}\left|\frac{\partial^{k} \varphi(x y)}{\partial(x y)^{k}}\right|^{2} d y\right\}^{1 / 2} \\
& \times\left\{\int_{-\infty}^{\infty}\left(1+y^{2}\right)\left|y^{k} B u(y)\right|^{2} d y\right\}^{1 / 2} .
\end{aligned}
$$

Note that $\left|\partial^{k} \varphi(x y) / \partial(x y)^{k}\right| \leq a$ for all $x, y \in \mathbf{R}^{1}$ and for all $k=0,1,2, \cdots, \alpha$. Here $a$ depends on $c$ and $\alpha$, i.e., on $c$ and $m$. Hence $\left|d^{k} v(x) / d x^{k}\right| \leq \sqrt{\pi a^{2}}|u|_{m}$. Therefore, $v \in \mathcal{B}^{\alpha}\left(\mathbf{R}^{1}\right)$ and $|v|_{\mathcal{B}^{\alpha}} \leq \sqrt{\pi a^{2}}|u|_{m}$.

Lemma 3.4. Let $c$ and $\beta$ be as in Theorem 1.2. Let $v$ be given by (3.1).

(A) $v / x^{\beta}$ is continuous on $\mathbf{R}^{1}$.

(B) $\quad v \in \mathcal{F}^{\beta}\left(\mathbf{R}^{1}\right)$ and $|v|_{\mathcal{F}^{\beta}} \leq b|u|_{m}$, where $b$ is a positive constant and depends on $m$ and $c$ only.

Proof. (A) By (3.1),

$$
\frac{v(x)}{x^{\beta}}=\int_{-\infty}^{\infty} \frac{\varphi(x y)}{(x y)^{\beta}} y^{\beta} B u(y) d y .
$$

Lemma 3.2 implies that $v$ is continuous on $\mathbf{R}^{1}$, and hence it suffices to show the continuity of $v / x^{\beta}$ at $x=0$.

The case where $c=2 k(k=1,2,3, \cdots)$ and $c \leq m-1$. Note that $\beta=c$. Lemma 2.5 implies that the integral

$$
\left[\frac{v}{x^{\beta}}\right]_{x=0}=\int_{-\infty}^{\infty} \frac{y^{2 k}}{2^{2 k+1 / 2} \Gamma(2 k+1 / 2)} B u(y) d y
$$

is well defined. Here $\Gamma$ denotes the gamma function. Then

$$
\frac{v(x)}{x^{\beta}}-\left[\frac{v}{x^{\beta}}\right]_{x=0}=\int_{-\infty}^{\infty} G(x y) y^{2 k} B u(y) d y,
$$


where $G(x y)=(x y)^{-2 k} \varphi(x y)-\left\{2^{2 k+1 / 2} \Gamma(2 k+1 / 2)\right\}^{-1}$. It follows from (2.1) that $\varphi /(x y)^{2 k}$ satisfies condition (i) of Lemma 3.2. Furthermore, since $|G(x y)| \leq$ constant $\cdot|x y|$, condition (ii) is also satisfied. Thus $v / x^{\beta}$ is continuous at $x=0$.

The other cases. By $(2.1), \varphi /(x y)^{\beta}$ satisfies condition (i) of Lemma 3.2. Note that $\left[\varphi /(x y)^{\beta}\right]_{x=0}=0$. It follows from (2.2) that

$$
\left|\frac{\varphi(x y)}{(x y)^{\beta}}\right| \leq \text { constant } \cdot|x y|^{c-\beta} \text {. }
$$

Thus condition (ii) is satisfied, and hence $v / x^{\beta}$ is continuous at $x=0$.

(B) From (3.3) we obtain that for $l=0,1,2, \cdots, \beta$,

$$
\begin{aligned}
\left|\frac{v(x)}{x^{l}}\right| \leq & \left\{\int_{-\infty}^{\infty} \frac{1}{1+y^{2}}\left|\frac{\varphi(x y)}{(x y)^{l}}\right|^{2} d y\right\}^{1 / 2} \\
& \times\left\{\int_{-\infty}^{\infty}\left(1+y^{2}\right)\left|y^{l} B u(y)\right|^{2} d y\right\}^{1 / 2} .
\end{aligned}
$$

Note that $\left|\varphi(x y) /(x y)^{l}\right| \leq b$ for all $x, y \in \mathbf{R}^{1}$ and for all $l=0,1,2, \cdots, \beta$. Here $b$ depends on $c$ and $\beta$, i.e., on $c$ and $m$. Hence $\left|v(x) / x^{l}\right| \leq \sqrt{\pi b^{2}}|u|_{m}$. Therefore, $v \in \mathcal{F}^{\beta}\left(\mathbf{R}^{1}\right)$ and $|v|_{\mathcal{F}^{\beta}} \leq \sqrt{\pi b^{2}}|u|_{m}$.

\section{Applications}

In this section we apply our main theorem, Theorem 1.2, to the Cauchy problems for partial differential equations with singular coefficients.

First we consider the Cauchy problem for the Schrödinger equation for a spinless, nonrelativistic quantum mechanical particle under the influence of the potential $k /\left(2 x^{2}\right):$

$$
\left\{\begin{array}{l}
i \frac{\partial u}{\partial t}=\frac{1}{2}\left(-\frac{\partial^{2}}{\partial x^{2}}+\frac{k}{x^{2}}\right) u, \quad x \in \mathbf{R}^{1} ; \\
u(0, x)=f(x), \quad x \in \mathbf{R}^{1} .
\end{array}\right.
$$

Let us recall that both $\mathcal{D}$ and $\mathcal{H}^{2}\left(\mathbf{R}^{1}\right)$ depend on the constant $c$. For a moment, we denote $\mathcal{D}$ and $\mathcal{H}^{2}\left(\mathbf{R}^{1}\right)$ by $\mathcal{D}_{c}$ and $\mathcal{H}_{c}^{2}\left(\mathbf{R}^{1}\right)$, respectively. Let $u_{+}=(u+R u) / 2$, $u_{-}=(u-R u) / 2$, where $R u(t, x)=u(t,-x)$. We interpret this equation in $L^{2}\left(\mathbf{R}^{1}\right)$ as

$$
i \frac{d u_{+}}{d t}=-\frac{1}{2}\left(\mathcal{D}_{c+1}\right)^{2} u_{+}, \quad u_{+}(0)=f_{+} \in \mathcal{H}_{c+1}^{2}\left(\mathbf{R}^{1}\right)
$$

and

$$
i \frac{d u_{-}}{d t}=-\frac{1}{2}\left(\mathcal{D}_{c}\right)^{2} u_{-}, \quad u_{-}(0)=f_{-} \in \mathcal{H}_{c}^{2}\left(\mathbf{R}^{1}\right),
$$

where $c>1$ and $c(c+1)=k$. By Lemma 2.4, both $\left(\mathcal{D}_{c+1}\right)^{2}$ and $\left(\mathcal{D}_{c}\right)^{2}$ are selfadjoint, and hence each of $i\left(\mathcal{D}_{c+1}\right)^{2} / 2$ and $i\left(\mathcal{D}_{c}\right)^{2} / 2$ generates a strongly continuous unitary group on $L^{2}\left(\mathbf{R}^{1}\right)$. Therefore,

$$
u_{+}(t, \cdot)=\exp \left\{\frac{i}{2} t\left(\mathcal{D}_{c+1}\right)^{2}\right\} f_{+} \quad \text { and } \quad u_{-}(t, \cdot)=\exp \left\{\frac{i}{2} t\left(\mathcal{D}_{c}\right)^{2}\right\} f_{-}
$$

are unique solutions of (4.1) and (4.2), respectively. We remark here that when $x \in \mathbf{R}^{N}$ and $k>N$, the self-adjointness of $-\triangle+k|x|^{-2}$ was proved by Sohr [7, Folgerung 2.4]. Okazawa [5, Theorem 6.8] improved this result and showed that 
$-\triangle+k|x|^{-2}$ with domain $H^{2}\left(\mathbf{R}^{N}\right) \cap D\left(|x|^{-2}\right)$ is self-adjoint for $k>-(N-4) N / 4$ and essentially self-adjoint for $k=-(N-4) N / 4$. Here $D\left(|x|^{-2}\right)=\{u(x)$ : $\left.u,|x|^{-2} u \in L^{2}\left(\mathbf{R}^{N}\right)\right\}$. See also Reed and Simon [6, Theorem X.11]. Let $k>2$ and let $H$ be the operator $H=2^{-1}\left\{-\partial^{2} / \partial x^{2}+k / x^{2}\right\}$ with domain $H^{2}\left(\mathbf{R}^{1}\right) \cap D\left(x^{-2}\right)$. Then

$$
v(t, \cdot)=\exp (-i t H) f, \quad f \in H^{2}\left(\mathbf{R}^{1}\right) \cap D\left(\frac{1}{x^{2}}\right),
$$

is a unique solution of the Schrödinger equation under consideration.

Proposition 4.1. Suppose $c>1$ and $c(c+1)=k$. Let $u_{+}$and $u_{-}$be the solutions of (4.1) and (4.2) respectively.

(A) $u_{+}(t, \cdot)+u_{-}(t, \cdot) \in \mathcal{B}^{1}\left(\mathbf{R}^{1}\right) \cap \mathcal{F}^{1}\left(\mathbf{R}^{1}\right)$.

(B) If $c>3 / 2, c(c+1)=k$ and $f \in H^{2}\left(\mathbf{R}^{1}\right) \cap D\left(x^{-2}\right)$, then $u_{+}(t, \cdot)+$ $u_{-}(t, \cdot)=v(t, \cdot)$, where $v$ is given by $(4.3)$.

Proof. Since $u_{+}(t, \cdot) \in \mathcal{H}_{c+1}^{2}\left(\mathbf{R}^{1}\right)$ and $u_{-}(t, \cdot) \in \mathcal{H}_{c}^{2}\left(\mathbf{R}^{1}\right)$, (A) immediately follows from Theorem 1.2.

(B) Note that the operator $-i \mathcal{D}_{c}$ is self-adjoint on the set $\left\{u \in H^{1}\left(\mathbf{R}^{1}\right): u / x \in\right.$ $\left.L^{2}\left(\mathbf{R}^{1}\right)\right\}$ when $c>1[8$, Theorem 1]. Then an elementary calculation gives

$$
\left(\left(\mathcal{D}_{c+1}\right)^{2} f_{+}, \phi\right)=\left(\left\{\frac{\partial^{2}}{\partial x^{2}}-\frac{k}{x^{2}}\right\} f_{+}, \phi\right)
$$

for $\phi \in C_{0}^{\infty}\left(\mathbf{R}^{1} \backslash\{0\}\right)$. Hence $\left(\mathcal{D}_{c+1}\right)^{2} f_{+}=\left(\partial^{2} / \partial x^{2}-k / x^{2}\right) f_{+}$. A similar argument gives $\left(\mathcal{D}_{c}\right)^{2} f_{-}=\left(\partial^{2} / \partial x^{2}-k / x^{2}\right) f_{-}$. Note again that $\mathcal{H}_{c}^{2}\left(\mathbf{R}^{1}\right)$ coincides with $H^{2}\left(\mathbf{R}^{1}\right) \cap D\left(x^{-2}\right)$ as long as $c>3 / 2$ [4]. The result follows.

Second we consider the Cauchy problem for the Schrödinger equation for a onedimensional harmonic oscillator governed by Wigner's commutation relations:

$$
\left\{\begin{array}{l}
i \frac{\partial u}{\partial t}=\frac{1}{2}\left\{-\frac{\partial^{2}}{\partial x^{2}}+\frac{c}{x^{2}}(c-R)+x^{2}\right. \\
u(0, x)=f(x), \quad x \in \mathbf{R}^{1} .
\end{array}\right.
$$

We interpret the Schrödinger equation in $L^{2}\left(\mathbf{R}^{1}\right)$ as

$$
i \frac{d u}{d t}=H u, \quad u(0)=f,
$$

where the Hamiltonian is given by $H=-\mathcal{D}^{2} / 2+x^{2} / 2$. From [3, Theorem 2.2] and [3, Proposition 4.2] we see that $(1 / 2) B^{*} y^{2} B+(1 / 2) x^{2}$ is self-adjoint on $\left\{B^{*} D\left(y^{2}\right)\right\} \cap D\left(x^{2}\right)$. Hence Definition 2.3 and Lemma 2.4 imply that $H$ is selfadjoint on $\mathcal{H}^{2}\left(\mathbf{R}^{1}\right) \cap D\left(x^{2}\right)$. Therefore, $-i H$ generates a strongly continuous unitary group on $L^{2}\left(\mathbf{R}^{1}\right)$. We thus see that

$$
u(t, \cdot)=\exp (-i t H) f, \quad f \in \mathcal{H}^{2}\left(\mathbf{R}^{1}\right) \cap D\left(x^{2}\right)
$$

is a unique solution of the Schrödinger equation (4.4). Note that the solution $u(t, \cdot)$ is in $\mathcal{H}^{2}\left(\mathbf{R}^{1}\right) \cap D\left(x^{2}\right)$. Theorem 1.2 thus implies the following.

Proposition 4.2. Let $c>1$ and let $u$ be the solution of (4.4). Then $u(t, \cdot) \in$ $\mathcal{B}^{1}\left(\mathbf{R}^{1}\right) \cap \mathcal{F}^{1}\left(\mathbf{R}^{1}\right)$. Moreover, $|u(t, \cdot)|_{\mathcal{B}^{1}} \leq a|u(t, \cdot)|_{2}$ and $|u(t, \cdot)|_{\mathcal{F}^{1}} \leq$ $b|u(t, \cdot)|_{2}$, where $a$ and $b$ are positive constants that depend on $c$ only. 
Finally we consider the Cauchy problem for the following equation of hyperbolic type:

$$
\left\{\begin{array}{l}
\frac{\partial^{2} u}{\partial t^{2}}=(-1)^{m+1}\left\{\frac{\partial^{2}}{\partial x^{2}}-\frac{c}{x^{2}}(c-R)\right\}^{m} u, \quad t \in \mathbf{R}^{1}, \quad x \in \mathbf{R}^{1} \\
u(0, x)=f(x), \quad \frac{\partial u}{\partial t}(0, x)=g(x), \quad x \in \mathbf{R}^{1} .
\end{array}\right.
$$

Here $m=1,2,3, \cdots$. We interpret this equation in $L^{2}\left(\mathbf{R}^{1}\right)$ as

$$
\frac{d^{2} u}{d t^{2}}=-(-i \mathcal{D})^{2 m} u, \quad u(0)=f, \quad \frac{d u}{d t}(0)=g .
$$

Suppose that $f \in \mathcal{H}^{2 m}\left(\mathbf{R}^{1}\right)$ and $g \in \mathcal{H}^{m}\left(\mathbf{R}^{1}\right)$. By Lemma $2.4(\mathrm{C})$ the operator $-i \mathcal{D}$ is self-adjoint, and so the Cauchy problem (4.5) is well-posed. See e.g. Goldstein $[1, \mathrm{p} .113]$. Hence the solution $u(t, \cdot)$ is in $\mathcal{H}^{2 m}\left(\mathbf{R}^{1}\right)$. Theorem 1.2 thus implies the following.

Proposition 4.3. Suppose $c>1, f \in \mathcal{H}^{2 m}\left(\mathbf{R}^{1}\right)$ and $g \in \mathcal{H}^{m}\left(\mathbf{R}^{1}\right)$. Let $u$ be the solution of (4.5). Then $u(t, \cdot) \in \mathcal{B}^{\alpha}\left(\mathbf{R}^{1}\right) \cap \mathcal{F}^{\beta}\left(\mathbf{R}^{1}\right)$, where

$$
\alpha= \begin{cases}2 m-1 & (c=2 k) \\ \min (2 m-1, c-1) & (c=2 k+1), \\ \min (2 m-1,[c]) & \text { (otherwise) }\end{cases}
$$

and

$$
\beta= \begin{cases}\min (2 m-1, c) & (c=2 k) \\ \min (2 m-1, c-1) & (c=2 k+1) \\ \min (2 m-1,[c]) & (\text { otherwise })\end{cases}
$$

with $k=1,2, \cdots$. Moreover,

$$
|u(t, \cdot)|_{\mathcal{B}^{\alpha}} \leq a|u(t, \cdot)|_{2 m} \quad \text { and } \quad|u(t, \cdot)|_{\mathcal{F}^{\beta}} \leq b|u(t, \cdot)|_{2 m},
$$

where $a$ and $b$ are positive constants that depend on $m$ and $c$ only.

\section{REFERENCES}

1. J. A. Goldstein, Semigroups of linear operators and applications, Oxford University Press, New York, 1985 / Clarendon Press, Oxford, 1985. MR 87c:47056

2. Y. Ohnuki and S. Kamefuchi, Quantum field theory and parastatistics, University of Tokyo Press, Tokyo, 1982 / Springer-Verlag, Berlin, Heidelberg and New York, 1982. MR 85b:81001

3. Y. Ohnuki and S. Watanabe, Self-adjointness of the operators in Wigner's commutation relations, J. Math. Phys. 33 (1992), 3653-3665. MR 93h:81065

4. Y. Ohnuki and S. Watanabe, Properties of the operators in Wigner's commutation relations (in preparation).

5. N. Okazawa, On the perturbation of linear operators in Banach and Hilbert spaces, J. Math. Soc. Japan 34 (1982), 677-701. MR 84i:47021

6. M. Reed and B. Simon, Methods of modern mathematical physics, vol. II, Fourier analysis, self-adjointness, Academic Press, New York, 1975. MR 58:12429b

7. H. Sohr, Über die Selbstadjungiertheit von Schrödinger- Operatoren, Math. Z. 160 (1978), 255-261. MR 80d:35040

8. M. Watanabe and S. Watanabe, Self-adjointness of the momentum operator with a singular term, Proc. Amer. Math. Soc. 107 (1989), 999-1004. MR 90g:81035

9. S. Watanabe, Sobolev type theorems for an operator with singularity, Proc. Amer. Math. Soc. (to appear). CMP 95:16 
10. E. P. Wigner, Do the equations of motion determine the quantum mechanical commutation relations ?, Phys. Rev. 77 (1950), 711-712. MR 11:706e

11. L. M. Yang, A note on the quantum rule of the harmonic oscillator, Phys. Rev. 84 (1951), 788-790. MR 13:804e

Department of Mathematics, Toyota National College of Technology, Eisei-cho 2-1, TOYOTA-SHI 471 , JAPAN 